\title{
Serum amyloid A mediates the inhibitory effect of Ganoderma lucidum polysaccharides on tumor cell adhesion to endothelial cells
}

\author{
YING-BO LI ${ }^{1}$, RUI WANG ${ }^{1}$, HONG-LI WU ${ }^{1}$, YU-HUA LI ${ }^{1}$, LI-JUN ZHONG $^{2}$, HE-MING YU $^{3}$ and XUE-JUN LI ${ }^{1}$ \\ ${ }^{1}$ Department of Pharmacology, School of Basic Medical Sciences and State Key Laboratory of Natural \\ and Biomimetic Drugs; ${ }^{2}$ Medical and Health Analytical Center, Peking University, Beijing 100083; \\ ${ }^{3}$ National Research Institute for Family Planning, Beijing 100081, China
}

Received March 18, 2008; Accepted May 9, 2008

DOI: 10.3892/or_00000041

\begin{abstract}
Ganoderma ludicum polysaccharides (GlPS) are the major bioactive composition of Ganoderma lucidum, a well-recognized oriental medical fungus. The published data have shown a complementary effect of GlPS in cancer therapy. The present study was designed to determine the anti-tumor efficacy of GlPS and the possible mechanism covering this effect. Murine Sarcoma 180 (S180) model was established, and GlPS administered orally for 10 days. On the 10th day, tumors were weighed to assess the inhibitory effect of GlPS and sera were collected for proteomic analysis and in vitro study. The in vivo results demonstrated that 25,50 and $100 \mathrm{mg} / \mathrm{kg}$ GlPS inhibited S180 growth by 32.67, 44.80 and $45.24 \%$, respectively $(\mathrm{P}<0.01)$. Proteomic study revealed marked protein changes after the process of treatment. Three significantly changed proteins were identified by ESI-QTOF-MS and database search indicated that they were haptoblobin, apolipoprotein A-II and serum amyloid A (SAA), respectively. Additionally, the expression change of SAA was confirmed by both Western blot and RT-PCR. The adhesion assay showed that GlPS-treated sera dramatically inhibited the adhesion ability of human prostate carcinoma (PC-3M) cells to human umbilical cord vascular endothelial cells (HUVECs), and this effect partially recovered after immunodepletion by the antibody against SAA. Collectively, these results suggest that GlPS inhibited the tumor growth and tumor cell adhesion to HUVECs via up-regulation of SAA protein expression.
\end{abstract}

Correspondence to: Dr Xue-Jun Li, Department of Pharmacology, School of Basic Medical Sciences and State Key Laboratory of Natural and Biomimetic Drugs, Peking University, Beijing 100083, China

E-mail: xjli@bjmu.edu.cn

Key words: Ganoderma lucidum (Leyss. ex Fr.) Karst, polysaccharides, anti-tumor, two dimensional gel electrophoresis, mass spectrometry, serum amyloid A, adhesion

\section{Introduction}

Ganoderma lucidum (Leyss. ex Fr.) Karst, also known as 'Lingzhi' in China, has a long history of medical use in Asian countries. This medical mushroom, which was used to promote health and longevity in the past, has been recently shown to have therapeutic effects on many diseases, including neurasthenia, insomnia, nephritis, hepatitides, diabetes and cardiovascular disease (1-5). Especially, it shows prominent anti-tumor actions. In the 70s, T. Sasaki uncovered the antitumor effect of polysaccharides from Ganoderma applanatum (another species of Ganoderma) for the first time (6). From then on, both in vivo and in vitro research on the anti-tumor activity of Ganoderma lucidum have been carried out, supporting its application for cancer treatment and prevention. In addition, Ganoderma lucidum exhibited adjuvant role in combination with radiotherapy or chemotherapy. It can enhance the anti-tumor effect of chemotherapeutics. According to these reports, the main anti-tumor components in Ganoderma lucidum are polysaccharides (7) and triterpenoids (4), which are the main biological compounds of water extracts and alcohol extracts.

Because one of the effective ways to develop new drugs from bioactive compounds of natural products is to find the potential protein targets, the study on the molecular mechanisms of anti-tumor effect of GlPS has received much attention. Previous studies proposed that the cancer prevention activity of GlPS were carried out mainly through immunomodulation (7). However, some recent studies suggested other potential mechanisms, such as anti-angiogenesis (8), inhibition of tumor cell motility (9), induction of apoptosis (10), induction of phase II-metabolizing enzyme (11) and antimutagenic activities (12). Nevertheless, the mechanisms of anti-tumor activities of GlPS remain obscure. In order to better understand the anti-tumor mechanisms of GlPS, twodimensional gel electrophoresis analysis was adopted in this study. Through analyzing global protein profile in GlPStreated and control S180 mice coupling with mass spectrometry, we attempted to elucidate the possible anti-tumor mechanisms and find potential protein targets of GlPS. We observed the possible functions of one dramatically changed protein by cell-based research and compared the effects of 
GlPS-treated sera on the tumor cell adhesion ability to HUVECs with and without depleting of this protein. These findings provide preliminary evidence for a novel mechanism by which GlPS exerts its anti-tumor effect.

\section{Materials and methods}

Animals and drugs. Inbred male BALB/c mice weighing 17$22 \mathrm{~g}$ were purchased from the Department of Experimental Animals, Peking University Health Science Center, Beijing, China. All procedures were in accordance to the Institute Ethics Committee for Experimental Use of Animal. Ganoderma lucidum polysaccharides (GlPS) are gift from Professor Zhi-Bin Lin, Department of Pharmacology, Peking University Health Science Center. Ganoderma lucidum (Leyss. ex Fr.) Karst was collected in Fujian Province, China. The fruit body of Ganoderma lucidum (Leyss. ex Fr.) Karst was authenticated by Professor Xiao-Lan Mao, Institute of Microbiology of Chinese Academy of Science. GlPS was extracted by hot water from the fruiting body of Ganoderma lucidum (Leyss. ex Fr.) Karst, followed by ethanol precipitation, reserve dialysis and protein depletion. The yield of GlPS was $0.82 \%(w / w)$ in terms of the fruiting body of Ganoderma lucidum. It is a polysaccharide peptide with a molecular weight of 584900 and has 17 amino acids. The ratio of polysaccharides to peptides is $93.51: 6.49 \%$. The polysaccharides consist of rhamnose, xylose, fructose, galactose, mannose and glucose with molar ratios of 0.793:0.964: 2.944:0.167:0.384:7.94 and are linked by h-glycosidic linkages. It is a hazel-colored and water-soluble powder (4).

Sample preparation. Murine Sarcoma 180 (S180) cells were purchased from Beijing Tumor Institute and maintained by weekly transplantation of the tumor cells into the peritoneal cavity of mice. S180 cells ( $2 \times 10^{6} /$ mouse) were injected into the axillary fossa of the right foreleg. On the second day after inoculation, mice were separated into 4 groups $(n=10)$ to receive saline, or $25,50,100 \mathrm{mg} / \mathrm{kg}$ GlPS dissolved in saline orally once a day. On the 10th day after drug treatment, the mice were sacrificed and tumors were excised and weighed. At the same time, the serum samples and liver samples were separated and stored at $-70^{\circ} \mathrm{C}$ until use.

Cell culture. Human umbilical cord vascular endothelial cells (HUVECs) were isolated by $1 \%$ collagenase (Invitrogen, USA) from human umbilical cords, and were cultured in M199 (Gibco, USA) supplemented with $20 \%$ heat-inactivated fetal bovine serum (FBS, Gibco), $40 \mu \mathrm{g} / \mathrm{ml}$ endothelial cell growth supplement (ECGS, purchased from China-Japan Friend Hospital), $100 \mathrm{U} / \mathrm{ml}$ penicillin, $100 \mathrm{U} / \mathrm{ml}$ streptomycin, $50 \mu \mathrm{g} /$ $\mathrm{ml}$ heparin and $300 \mu \mathrm{g} / \mathrm{ml} \mathrm{L}$-glutamine. HUVECs were identified by the typical polygonal morphology and by detecting their immunoreactivity of factor-VIII related antigens. Human prostate carcinoma cells (PC-3M) were cultured in RPMI-1640 (Gibco) supplemented with $10 \%$ fetal bovine serum, $100 \mathrm{U} / \mathrm{ml}$ penicillin and $100 \mu \mathrm{g} / \mathrm{ml}$ streptomycin.

Two dimensional gel electrophoresis and image analysis. The protein concentration of serum samples was measured by Bradford method. Serum protein $(1 \mathrm{mg})$ was dissolved in the rehydration buffer ( $8 \mathrm{M}$ urea, 4\% CHAPS, $65 \mathrm{mM}$ DTT, $0.2 \%$ Bio-Lyte, $0.001 \%$ bromochlorphenol blue) to a final volume of $300 \mu 1$. This mixture was then applied to twodimensional electrophoresis. The first dimension isoelectric focusing (IEF) was performed using $18 \mathrm{~cm}, \mathrm{pH} 3-10$, linear immobilized pH gradients gel (IPG) strips (Amersham Pharmacea Biotech, USA). The IPG strips were rehydrated for $16 \mathrm{~h}$ at $20^{\circ} \mathrm{C}$ by placing the strips gel-side-down and covered with mineral oil. Then, IEF was performed at $20^{\circ} \mathrm{C}$ in following steps: $250 \mathrm{~V}$ linear for $0.5 \mathrm{~h}, 1000 \mathrm{~V}$ gradient for $1 \mathrm{~h}, 8000 \mathrm{~V}$ linear for $5 \mathrm{~h}, 8000 \mathrm{~V}$ gradient until $60000 \mathrm{Vh}$. After IEF, the IPG strips were equilibrated in buffer containing $6 \mathrm{M}$ urea, 20\% glycerol, $0.375 \mathrm{M}$ Tris- $\mathrm{HCl}(\mathrm{pH} 8.8), 2 \%$ SDS and $20 \mathrm{mg} / \mathrm{ml} \mathrm{DTT}$ for $15 \mathrm{~min}$, and then for another $15 \mathrm{~min}$ in the same buffer with $25 \mathrm{mg} / \mathrm{ml}$ iodoacetamide replacing DTT. Then, the second dimension was proceeded on $12 \%$ SDS-PAGE gels at $10 \mathrm{~mA} / \mathrm{gel}$ for $30 \mathrm{~min}$ followed by $20 \mathrm{~mA} / \mathrm{gel}$ until completion. Gels were stained with Coomassie blue or silver. The experimental procedures were repeated more than three times. Then, $2 \mathrm{D}$ gels were analyzed using the PDQuest software (Bio-Rad) according to the protocols provided by the manufacturer. All gel spots that detected as significantly changed were then highlighted and checked manually to prevent any artifacts due to gel distortions, inappropriately matched or mistakenly detected spots.

Protein identification by ESI-Q-TOF-MS/MS. The selected protein spots were excised from the gels for decolorization, in-gel digestion and MS analysis. Electrospray quadrupole/ time-of-flight mass spectrometry (ES-Q-TOF-MS, Waters, USA) was performed to identify the selected protein spots. The peptide mixture was carried out on a Waters Capillary liquid chromatography system including three pumps A, B and $\mathrm{C}$ (Waters). Fused silica tubing $(75 \mu \mathrm{m} \times 100 \mathrm{~mm})$ packed with symmetry $300^{\mathrm{TM}} \mathrm{C}_{18}, 3.5 \mu \mathrm{m}$ spherical particles with pore diameter $100 \AA$ (Waters) The flow rate was set at $2.5 \mu \mathrm{l} / \mathrm{min}$. Samples were injected at a flow rate of $20 \mu \mathrm{l} /$ min with pump $C$ and salts were removed on the precolumn of $(0.35 \times 5 \mathrm{~mm})$ packed with symmetry $300^{\mathrm{TM}} \mathrm{C} 18,3.5 \mu \mathrm{m}$ spherical particles with pore diameter $100 \AA$ (Waters). The precolumn was connected in the 10-port switching valve, and switched to the analytical column after the sample was desalted. Mobile phase A consisted of water/ACN (95/5, v/v) with 0.1 FA. Mobile phase B consisted of water/ACN (5/95, $\mathrm{v} / \mathrm{v}$ ) with $0.1 \mathrm{FA}$. The separation was performed by running an onlinear gradient: $3 \% \mathrm{~B}$, for 0.1-3.5 min for injection; $5-40 \% \mathrm{~B}$, for 3.5-40 $\mathrm{min}$; 40-60\% $\mathrm{B}$, for 40-60 min; 60$90 \% \mathrm{~B}$, for $60-65 \mathrm{~min}$; $90-5 \% \mathrm{~B}$, for $65-70 \mathrm{~min}$; $5-5 \% \mathrm{~B}$, for 70-90 min. The Cap LC is coupled on-line with a Q-TOF Ultima Global mass spectrometer (Waters) for detection and protein identification. Peptide mixtures were dissolved with $2 \mu 10.1 \%$ FA and inject by the atmosphere on the precolumn using a Cap LC system. Peptides were directly eluted into a Q-TOF mass spectrometer (Q-TOF Ultima Global mass spectrometer; Waters) at $250 \mathrm{nl} / \mathrm{min}$ on the analytical column. After being processed with Mass Lynx 4.0, the data resulting from the MS/MS analysis were applied for identification with MASCOT search engine (http://www.matrixsciencn. com). 
Western blot analysis. A sample consisting of $80 \mu \mathrm{g}$ of protein was separated by $15 \%$ polyacrylamide gels at $150 \mathrm{~V}$ constant power. Protein was then transferred electrophoretically at $200 \mathrm{~mA}$ to a polyvinylidene difluoride (PVDF) immobilion-P membrane $(0.22 \mu \mathrm{m}$ pore size; Millipore Corp., USA) in a transfer buffer ( $\mathrm{pH} 8.3$ ) composed of $25 \mathrm{mM}$ Tris- $\mathrm{HCl}, 192 \mathrm{mM}$ glycine and $20 \%$ methanol at $4^{\circ} \mathrm{C}$ for $2 \mathrm{~h}$. The membranes were blocked with Tris-buffered saline (TBS, $100 \mathrm{mM}$ Tris- $\mathrm{HCl}$ and $0.9 \% \mathrm{NaCl}, \mathrm{pH} 7.5$ ) containing $5 \%$ nonfat milk for $1 \mathrm{~h}$ at room temperature, and subsequently incubated with anti-SAA antibody (Biosource, USA) at a 1:1000 dilution at $4^{\circ} \mathrm{C}$ overnight. After three washes in TBS containing $0.1 \%$ Tween-20 (TBS-T), the membranes were incubated with 1:1000 HRP-conjugated secondary antibody with $5 \%$ non-fat milk for $1 \mathrm{~h}$ and visualized using the chemiluminescent ECL reagent.

Semi-quantitative RT-PCR analysis. For semi-quantitative reverse transcriptase-polymerse chain reaction (RT-PCR) analysis, total-RNAs were extracted from the liver samples of each group. The RNA concentration was determined by measuring the absorbance at $260 \mathrm{~nm}$, and its quality monitored by $0.8 \%$ agarose gel electrophoresis. Reverse transcription was carried out at $37^{\circ} \mathrm{C}$ for $60 \mathrm{~min}$, followed by $95^{\circ} \mathrm{C}$ for $2 \mathrm{~min}$ and first round PCR for $95^{\circ} \mathrm{C}$ for $30 \mathrm{sec}, 55^{\circ} \mathrm{C}$ for $30 \mathrm{sec}$, $72^{\circ} \mathrm{C}$ for $30 \mathrm{sec}$ and final extension at $72^{\circ} \mathrm{C}$ for $5 \mathrm{~min}$. Primers used in this study were as follows. SAA (5'-GCCTGGTCT TCTGCTCCCTG-3' and 5'-CCACTGCGGCCATGTCTG TT-3') and B-actin (5'-ATCATGTTTGAGACCTTCAACA-3' and 5'-CATCTCTTGCTCGAAGTCCA-3'). mRNA levels of $B$-actin were used to normalized the amount of RNA.

Cell adhesion assay. PC-3M cells were cultured in RPMI1640 supplemented with $100 \mathrm{mg} / \mathrm{l} \mathrm{CFDA}$ (Sigma, USA) for $30 \mathrm{~min}$ at $37^{\circ} \mathrm{C}$, then washed three times by PBS to remove the residue fluorescent dye. Cells viability was examined by trypan blue staining method and was not affected by this protocol.

HUVECs were cultured in 96-wells culture plate until confluence. Then the medium with control sera and GlPStreated sera $(25,50,100 \mathrm{mg} / \mathrm{kg})$ were changed respectively and cultured for $48 \mathrm{~h}$. And then the medium was removed, CFDA-labeled tumor cells (104/well) were added to 96-wells culture plate, and cocultured with HUVECs for $30 \mathrm{~min}$ at $37^{\circ} \mathrm{C}$. After cells were washed by PBS for three times, the number of adherent tumor cells was counted under fluorescent microscope in five fields each well (13).

Immunodepletion of SAA. Control sera and GlPS-treated sera were incubated with anti-human SAA monoclonal antibody (Biosource; 1:50 dilution) for $1 \mathrm{~h}$ at $4^{\circ} \mathrm{C}$, and then incubated with protein A-Agrose (Sigma, USA) for $12 \mathrm{~h}$ on a roller system at $4^{\circ} \mathrm{C}$. After centrifugalization at $15000 \mathrm{rpm}$ for $10 \mathrm{~min}$, the protein A-Agrose was removed. Then the immunodepleted sera were used for the adhesion assay.

Statistical analysis. Data were analyzed by one-way analysis of variance (ANOVA), followed by Dunnett's test (SPSS 10.0 software). Results are presented as means \pm SD. $\mathrm{P}<0.05$ was considered to be statistically significant.
Table I. The inhibitory effect of GlPS after oral treatment for 10 days on the growth of Sarcoma $180 \mathrm{in} \mathrm{Balb/c} \mathrm{mice.}$

\begin{tabular}{lcccc}
\hline Group & $\begin{array}{c}\text { Dose } \\
(\mathrm{mg} / \mathrm{kg})\end{array}$ & $\begin{array}{c}\text { Body } \\
\text { weight }(\mathrm{g})\end{array}$ & $\begin{array}{c}\text { Tumor } \\
\text { weight }(\mathrm{g})\end{array}$ & $\begin{array}{c}\text { Inhibitory } \\
\text { rate }(\%)\end{array}$ \\
\hline Control & - & $18.9 \pm 1.7$ & $1.10 \pm 0.11$ & - \\
& 25 & $19.4 \pm 1.5$ & $0.76 \pm 0.14^{\mathrm{a}}$ & 32.67 \\
GlPS & 50 & $19.3 \pm 1.2$ & $0.61 \pm 0.12^{\mathrm{a}}$ & 44.80 \\
& 100 & $18.2 \pm 1.4$ & $0.58 \pm 0.16^{\mathrm{a}}$ & 45.24 \\
\hline
\end{tabular}

Calculation of the inhibition rate based on the following formula: tumor inhibition rate (\%) [(average tumor weight of control group average tumor weight of GlPS group)/average tumor weight of control group] $x 100 \%$. Values are mean $\pm \mathrm{SD}, \mathrm{n}=6$. ${ }^{\mathrm{a}} \mathrm{P}<0.01$ vs control group. GIPS, Ganoderma lucidum polysaccharid.

\section{Results}

Inhibitory effect of GlPS on tumor growth. As shown in Table I, after the oral administration of 25,50 and $100 \mathrm{mg} / \mathrm{kg}$ GlPS for 10 contiguous days, the growth of tumor was inhibited by GlPS in S180 mouse model, and the tumor inhibition rate was $32.67,44.80$ and $45.24 \%$, respectively, which was identical with published studies by other groups.

$2 D$ gel electrophoresis and ES-Q-TOF-MS analysis on the sera of GIPS-treated and control mice bearing murine sarcoma 180 tumors. The serum samples from the control group and GlPS-treated groups were analyzed by 2D gel electrophoresis. Comparing the protein profiles by PDQuest software analysis, over 500 serum protein spots were recognized in each gel. Among them, three protein spots which were well separated (Fig. 1) were cut off from Coomassie blue-stained gels and subjected to ES-Q-TOF-MS analysis. Combining with database search, the spots were identified as haptoblobin, apolipoprotein A-II and serum amyloid A, respectively. The data are summarized in Table II. The MS/MS results of the three identified proteins are shown in Fig. 2.

Validation of differentially expressed protein by Western blot analysis. Through functional exploring of the three identified proteins, serum amyloid A (SAA) was chosen for further study. To confirm the identification and expression change of SAA, sera were analyzed by Western blot for validation. As shown in Fig. 2C, the serum level of SAA was increased in S180 mouse model after the treatment of GlPS, and this result was consistent with the $2 \mathrm{D}$ gel electrophoretic analysis.

Validation of differentially expressed protein by semiquantitative RT-PCR analysis. In previous research, SAA protein in the serum was suggested to be of liver origin (14), therefore, the SAA levels in liver samples were examined by semi-quantitative RT-PCR analysis for further validation. 
(A)

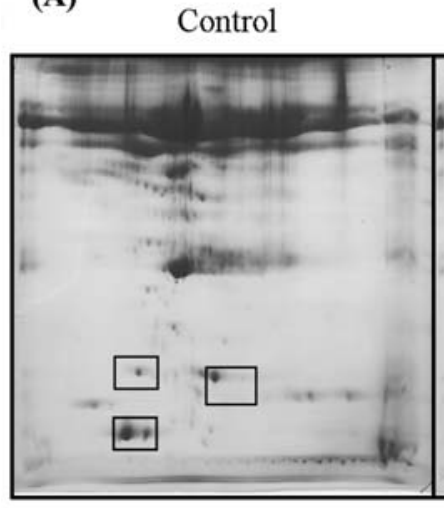

$\mathrm{pH} 3$

Control
GIPS-treated

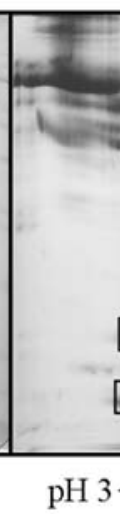

(B)

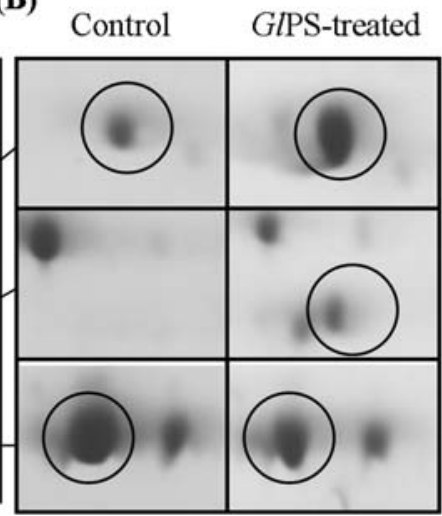

(C)

Control GlPS-treated

SAA

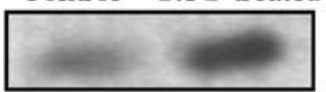

Figure 1. (A) Profiles of serum proteins from mice in control group and GIPS-treated group by 2D. The IEF was for pH 3-10. (B) The enlarged local images of proteins in the 2D gels. Proteins in serum from mice inoculated with Sarcoma 180 subjected to identification by MS are marked by circles. (C) Western blot analysis of SAA expression in serum of S180 mouse model with or without the treatment of GlPS. SAA level in serum increased after the treatment of GlPS. Control, serum sample from mice in control group; GlPS-treated, serum sample from mice in $100 \mathrm{mg} / \mathrm{kg}$ GlPS-treated group.

Table II. ES-Q-TOF-MS identified proteins in control serum vs GlPS-treated serum. The Swiss-Prot accession number, protein name, the $\%$ coverage of analyzed peptides, the score from Mascot searches and $M_{r}$ are shown for each protein.

\begin{tabular}{cllcrrr}
\hline Spot no. & Accession no. & \multicolumn{1}{c}{ Protein name } & \% coverage & Score & PI & $M_{r}(\mathrm{Da})$ \\
\hline 1 & gi8850219 & Haptoglobin [Mus musculus] & 4 & 59 & 5.88 & 38727 \\
2 & gi7304897 & Apolipoprotein A-II [Mus musculus] & 25 & 212 & 4.9 & 11312 \\
3 & gi200904 & Serum amyloid A & 34 & 355 & 6.21 & 12876 \\
\hline
\end{tabular}

The results are shown in Fig. 3. The mRNA level of SAA was up-regulated in a dose-dependent manner. The pattern of change was in agreement with $2 \mathrm{D}$ results, so the results of RT-PCR analysis confirmed the reliability of comparative proteomic study.

Inhibition effect of GlPS-treated sera on the adhesion ability of PC-3M to HUVECs. The effect of GlPS-treated sera on the metastatic adhesion function of malignant cells was evaluated by testing its effect on PC-3M cell adhesion to HUVCEs monolayers. It is clear from Fig. 4 that 25,50 and $100 \mathrm{mg} / \mathrm{kg}$ GlPS-treated sera can significantly inhibit the attachment of PC-3M cells to HUVECs, respectively $(\mathrm{P}<0.01)$. Meanwhile, examined by MTT method, no cytotoxic effect on HUVECs was seen at the same concentrations (data not show).

SAA-immunodepleted sera partially abolish the inhibition ability of GIPS-treated sera on the adhesion of PC-3M to HUVECs. After removal of SAA by immunodepletion with anti-SAA antibody in control and GlPS-treated sera, the cell adhesion assay was carried out using immunodepleted sera. As shown in Fig. 5. SAA-depleted GlPS-treated sera (25 and $50 \mathrm{mg} / \mathrm{ml}$ ) showed no inhibition effect on adhesion ability of PC-3M cells to HUVECs comparing with the control group, but $100 \mathrm{mg} / \mathrm{ml}$ SAA-depleted GlPS-treated serum still exerted significant inhibition ability. The inhibitory effect of GlPStreated sera on adhesion ability of PC-3M to HUVECs was partially abolished after the depletion of SAA.

\section{Discussion}

The anti-tumor activity of GlPS has been widely studied both in vitro and in vivo. In agreement with these findings, our results listed in Table I revealed that GlPS inhibited the growth of Sarcoma 180 by $32.67,44.80$ and $45.24 \%$, respectively.

Cancer is a multisteps and complex process that attributes to protein changes at functional level. The proteins change as the components or the ultimate effectors in the altered signaling pathways lead to cancer survival, growth, invasion and metastasis (15). Therefore, analyzing protein changes on tumor tissue or sera in animals bearing cancer will be helpful in uncovering the mechanisms of this pathological process and discovering anti-tumor drugs. As a high-throughput approach of mapping and analyzing protein profiles, proteomic methods show great advantages in cancer research. It offers scientists a feasible approach to explore global protein alterations rather than limited ones. The 
(A)

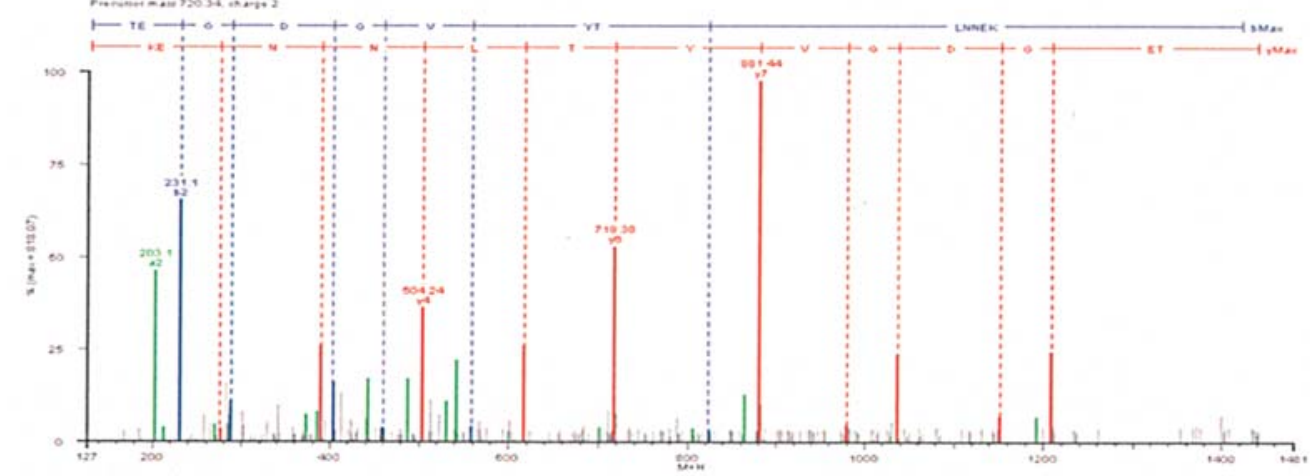

(B)

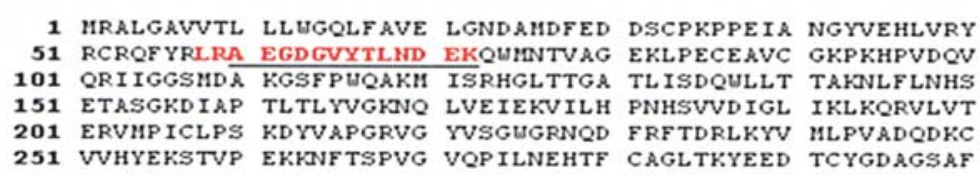

(C)

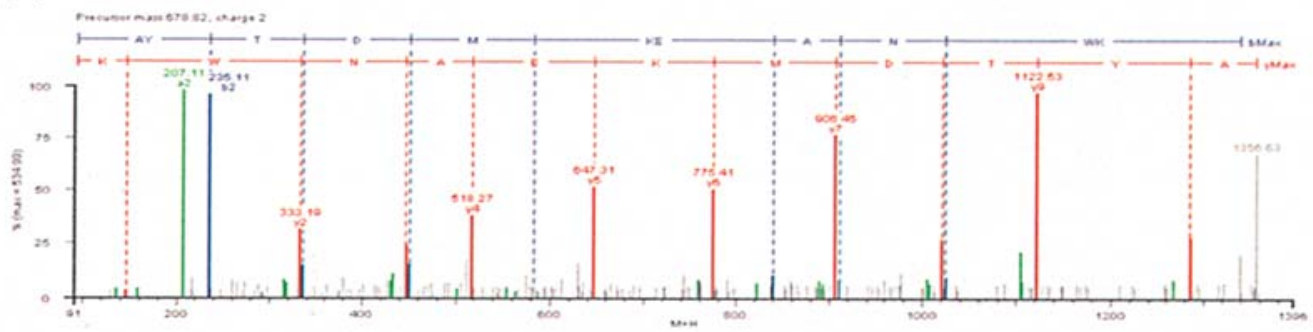

(D)

$\begin{aligned} 1 & \text { FCSLLLGVCH GGFFSFVHEA FQGAGDMURA YTDINEAMHK ISDKXFHARG } \\ 51 & \text { NYDAAQRGPG GVUAEKISD GREAFOEFFG RGEDTIADO EARHGRGK }\end{aligned}$ 51
101
1

(E)

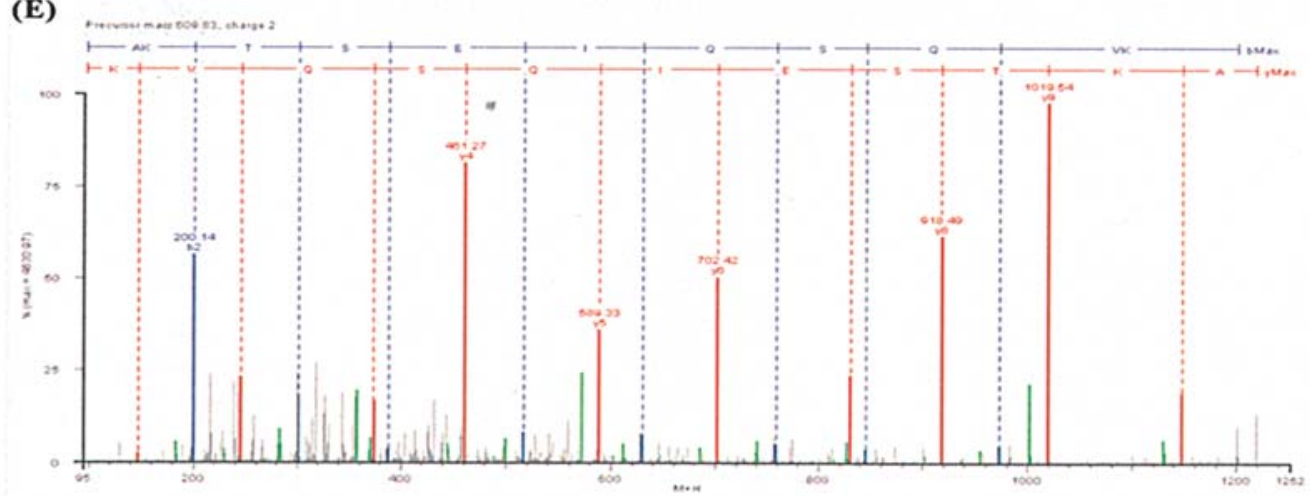

(F)

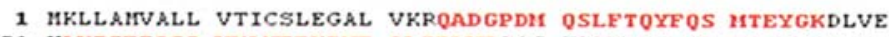
51 KAKTSEIOSO VKAYFEKTHE QLTPLVRSAG TSLVNFFSSL INUEEKPAPA 101 A.

Figure 2. ESI-Q-TOF-MS analysis of differentially expressed proteins. (A) The amino acid sequence of a double charged peptide with $m / z$. 720.34 was identified as TEGDGVYTLNNEK from mass differences in the y-fragment ions series, and matched with residues 60-72 of haptoglobin [Mus musculus]. (B) Protein sequence of haptoglobin precursor is shown. Matched MS/MS fragmentation is underlined. (C) The amino acid sequence of a double charged peptide with $m / z$, 609.832 was identified as AKTSEIQSQVK from mass differences in the y-fragment ions series, and matched with residues 52-62 of apolipoprotein A-II [Mus musculus]. (D) Protein sequence of apolipoprotein A-II is shown. Matched MS/MS fragmentation is underlined. (E) The amino acid sequence of a double charged peptide with $\mathrm{m} / \mathrm{z} 678.82$ was identified as AYTDMKEANWK from mass differences in the y-fragment ions series, and matched with residues 30-40 of serum amyloid A. (F) Protein sequence of serum amyloid A is shown. Matched MS/MS fragmentation is underlined.

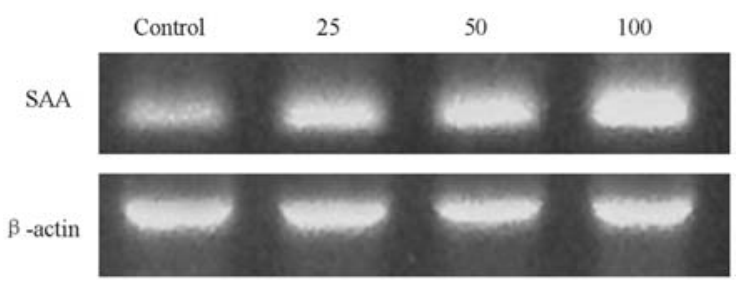

Figure 3. Semi-quantitative RT-PCR analysis of SAA mRNA expression in liver samples of S180 mouse model with or without the treatment of different dose of GlPS. Total-RNA was extracted and amplified by RT-PCR using gene-specific primers. Control, liver sample of mice in control group; 25,50 and 100 , liver samples of mice in GlPS-treated groups $(25,50$ and $100 \mathrm{mg} / \mathrm{kg}$, respectively); SAA PR-PCR product length, $304 \mathrm{bp}$; $\beta$-actin RTPCR product length, $540 \mathrm{bp}$. 

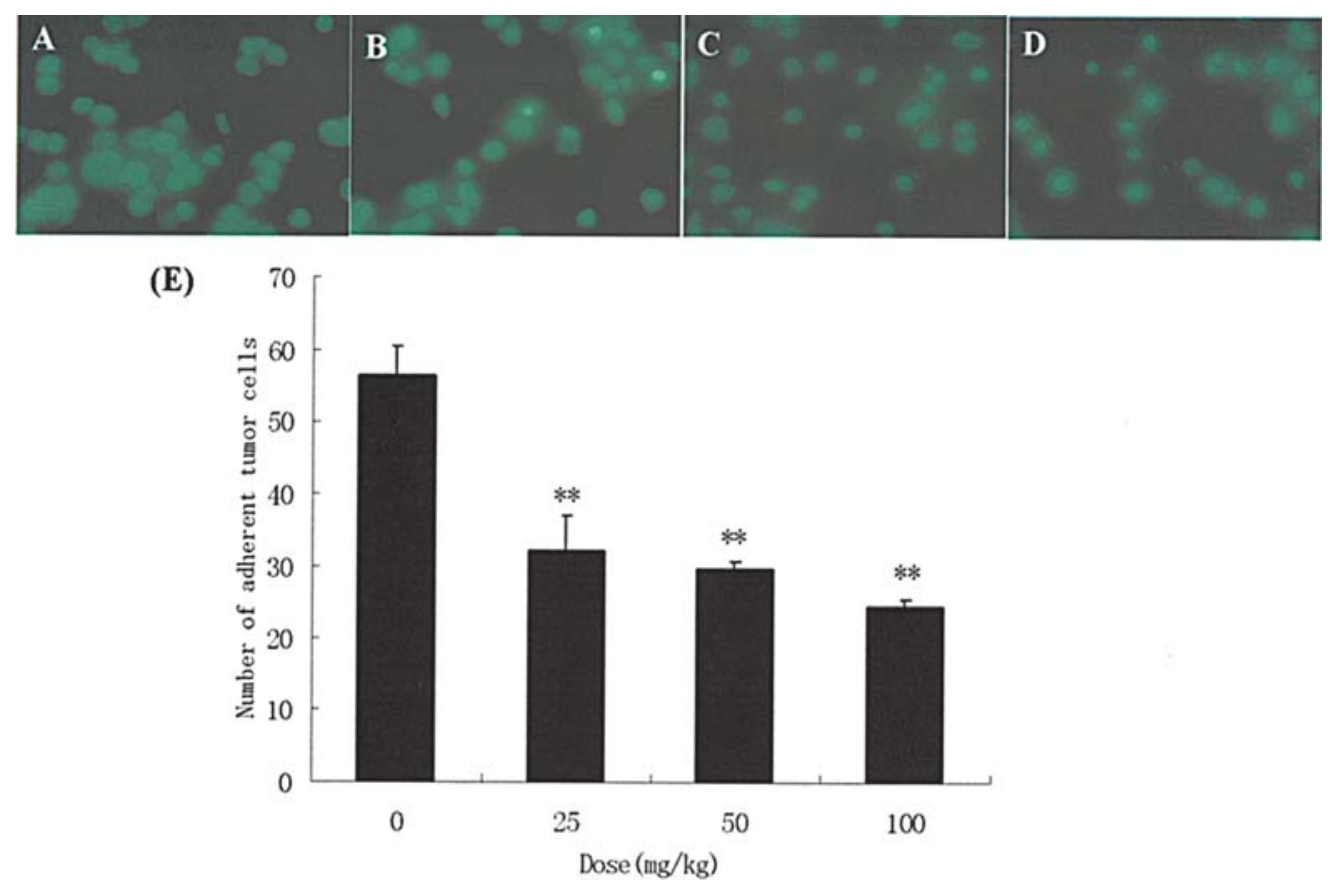

Figure 4. The inhibitory effect of GlPS-treated serum on the adhesion ability of PC-3M cells to HUVECs. The adhesion assay was performed with control serum and $25,50,100 \mathrm{mg} / \mathrm{kg}$ GlPS-treated serum. Adhesion ability of PC-3M cells to HUVECs was significantly inhibited by GlPS-treated serum. (A) Control; (B) $25 \mathrm{mg} / \mathrm{kg}$ GlPS-treated serum; (C) $50 \mathrm{mg} / \mathrm{kg}$ GlPS-treated serum; (D) $100 \mathrm{mg} / \mathrm{kg}$ GlPS-treated serum. Magnification x400. (E) Inhibition of PC-3M adhesion to HUVECs by GlPS-treated serum. $\mathrm{n}=5$, mean $\pm \mathrm{SD} .{ }^{* *} \mathrm{P}<0.01$ vs control.

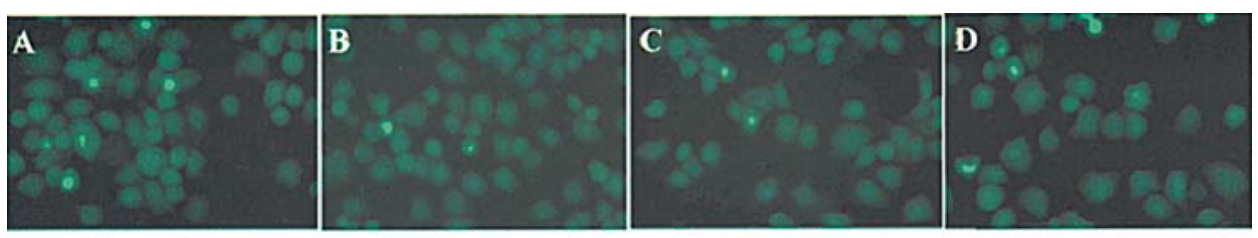

(E)

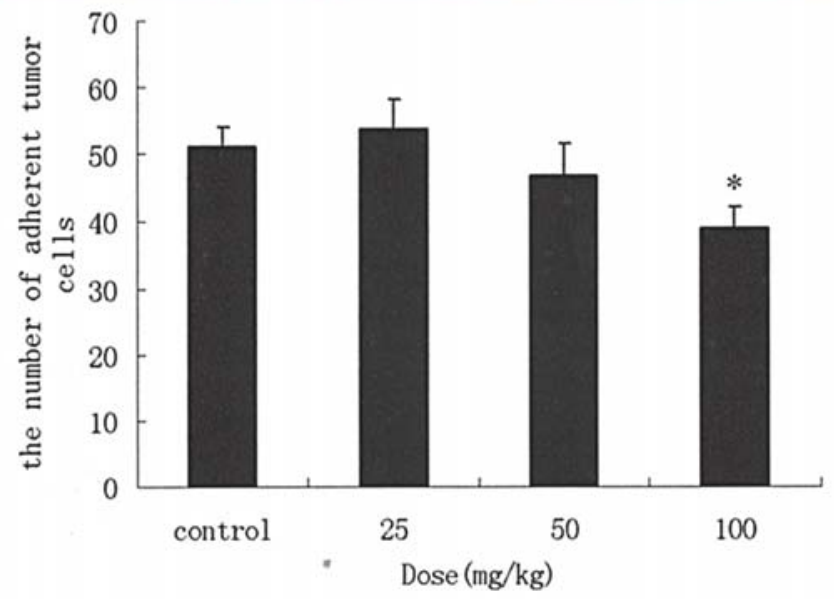

Figure 5. The effect of SAA-depleted GlPS-treated serum on the adhesion ability of PC-3M cells to HUVECs. SAA protein in GIPS-treated serum was depleted by immunodepletion assay using anti-SAA antibody. Then the adhesion assay was carried out using these SAA-depleted GlPS-treated sera. (A) SAA-depleted control; (B) $25 \mathrm{mg} / \mathrm{kg}$ SAA-depleted GlPS-treated serum; (C) $50 \mathrm{mg} / \mathrm{kg}$ SAA-depleted GlPS-treated serum; (D) $100 \mathrm{mg} / \mathrm{kg}$ SAA-depleted GlPS-treated serum. Magnification x400. (E) The inhibitory effect of GlPS-treated serum was partially abolished after the depletion of SAA. n=5, mean \pm SD. ${ }^{*} \mathrm{P}<0.05$ vs control.

differential expression patterns of these proteins would help us foster a better understanding of the pathophysio-logical molecular mechanisms of cancer (16). In addition, traditional medicines usually exert their effects through multitargets and multiapproaches, which might involve several protein expression changes and modifications. Therefore, in our study, proteomic methods were applied in the GlPS study, hoping to elucidate the comprehensive serum protein expression after the drug treatment and to find the potential proteins related to anti-tumor mechanism of GlPS. 
In this study, serum proteins were separated by their isoelectric points in the first IEF dimension, and then by their molecular mass using sodium dodecyl sulfate polyacrylamide gel electrophoresis (SDS-PAGE) in the second dimension. Comparing the proteins present in sera of control and GlPStreated mice by computer-assisted comparative analysis, marked changes in protein profiles were discovered. Three protein spots showed relatively greater changes. These spots were in well separated areas and were excised from gels for MS analysis. The results revealed that these proteins were SAA, haptoglobin, which were up-regulated after the treatment of GIPS, and apolipoprotein A-II, which was down-regulated after the treatment.

Through function exploring, SAA was chosen for further study among the three proteins because of its important role in the process of cancer. SAA is one of the major acute-phase serum proteins, which appears at low concentration in health individuals but is increased up to 1000 -fold in response to inflammation or various malignancies (17-19), suggesting its beneficial role in host defense. As the precursor of amyloid A (AA), SAA is probably involved in the progression of secondary amyloidosis and AA-amyloidosis (20). In addition, it is an apolipoprotein of high-density lipoprotein (HDL) and reported to participate in cholesterol metabolism and transport (21). More importantly, recent studies demonstrated that several functions for the SAA could be essential in neoplasia, which mainly attribute to the effect on the tumor cell adhesion and migration. These functions included inhibiting malignant cell attachment to extracellular matrix (22), inducing the expression of enzymes degrading the $\operatorname{ECM}(17,23,24)$, and stimulating leukocytes recruitment $(7,25)$. In addition, it is regarded as the biomarker in several types of malignancies, such as gastric cancer (19), pancreatic cancer (26), non-small cell lung cancer (27) and nasopharyngeal cancer (28). According to these reports, we speculated that regulation of SAA expression might be one of the contributing factors to anti-tumor effects of GlPS.

Therefore, we used two different methods to test our hypothesis. Since adhesion of tumor cells to endothelial cells is a critical step in tumor metastasis, adhesion assay was chosen for the examination of the effect of SAA expression in the anti-tumor activity of GlPS. In the first approach, adhesion assay was performed with GlPS-treated serum and control serum. As shown in Fig. 4, the adhesion ability of PC-3M cells was significantly inhibited by GlPS-treated serum, in which the concentration of SAA is much higher than the control serum. In the second approach, we used SAA monoclonal antibody to immunodeplete the SAA protein in GlPS-treated sera and control serum, and then compared the adhesion abilities of tumor cells with immunodepleted sera, in all of which the SAA is absent. As shown in Fig. 5, the inhibition effect partially recovered after immunodepletion. Together, these results confirmed that up-regulation of SAA is one possible mechanism for GlPS to inhibit the tumor cell adhesion to endothelial cells, i.e., one possible anti-tumor mechanism of GlPS.

The mechanisms by which SAA protein inhibits the adhesion ability of tumor cells to endothelial cells remain unclear. One possible explanation may be related to the functional adhesion motifs of SAA. According to previous reports, some proteins containing the peptide Tyr-IIe-GlySer-Arg (YIGSR) and/or Arg-Gly-Asp (RGD) demonstrated inhibition activities in tumor cell invasion and metastasis, such as the multimeric YIGSR-containing peptide (Ac-Y16), and RGD-containing peptide (rhodostomin) (29-31). Both functional YIGSR-like and RGD-like motifs also present in the SAA protein (22). Therefore, it is plausible that both of the functional peptides for SAA were related to inhibit the adhesion of tumor cells to endothelial cells.

In addition, the dose of $100 \mathrm{mg} / \mathrm{kg}$ GlPS after removal of SAA still showed inhibition action of PC-3M cells adhesion to HUVECs, it suggested that other mechanisms might also be involved in this progression. Haptoblobin and apolipoprotein A-II, the other obviously changed proteins after the drug treatment, might be also related to the mechanism of the inhibition of GlPS on tumor growth. Further study on them is in progress in our laboratory.

In conclusion, this was the first investigation of the antitumor mechanism of GlPS by proteomic methods and some interesting results were obtained. We separated and identified three potential proteins, haptoblobin, apolipoprotein A-II and SAA from the serum sample of GlPS-treated mice by 2D gel electrophoresis combined with MS analysis. It was demonstrated that the SAA protein concentration in serum was correlated with inhibition of the adhesion ability of PC-3M cells to HUVECs. These results will be helpful in elucidating the anticancer mechanisms of Ganoderma lucidum polysaccharides.

\section{Acknowledgements}

This study was supported by the National Natural Science Foundation of China (No. 30171090, 30270528, 30572202, 30772571), 973 Program of the Ministry of Science and Technology in China (No. 2004CB518902), a research fund from The Ministry of Education of China (111 Project, No. B0700 and 985 Project).

\section{References}

1. Li YQ and Wang SF: Anti-hepatitis B activities of ganoderic acid from Ganoderma lucidum. Biotechnol Lett 28: 837-841, 2006.

2. Futrakul N, Panichakul T, Butthep P, Futrakul P, Jetanalin P, Patumraj P and Siriviriyakul P: Ganoderma lucidum suppresses endothelial cell cytotoxicity and proteinuria in persistent proteinuric focal segmental glomerulosclerosis (FSGS) nephrosis. Clin Hemorheol Microcirc 31: 267-272, 2004.

3. Tang W, Gao Y, Chen G, Gao H, Dai X, Ye J, Chan E, Huang M and Zhou S: A randomized, double-blind and placebo-controlled study of a Ganoderma lucidum polysaccharide extract in neurasthenia. J Med Food 8: 53-58, 2005.

4. Zhang $\mathrm{HN}$, He JH, Yuan $\mathrm{H}$ and Lin $\mathrm{ZB}$ : In vitro and in vivo protective effect of Ganoderma lucidum polysaccharide on alloxan-induced pancreatic islets damage. Life Sci 73: 2307-2319, 2003.

5. Woo CW, Man RY, Siow YL, Choy PC, Wan EW, Lau CS and $\mathrm{O} \mathrm{K}$ : Ganoderma lucidum inhibits inducible nitric oxide synthase expression in macrophages. Mol Cell Biochem 276: $165-171,2005$.

6. Sasaki T, Arai Y, Ikekawa T, Chihara G and Fukuoka F: Antitumor polysaccharides from some polyporaceae, Ganoderma applanatum (Pers.) Pat and Phellinus linteus (Berk. et Curt.) Aoshima. Chem Pharm Bull 19: 821-826, 1971.

7. Badolato R, Wang JM, Murphy WJ, Lloyd AR, Michiel DF, Bausserman LL, Kelvin DJ and Oppenheim JJ: Oppenheim, serum amyloid $\mathrm{A}$ is a chemoattractant: induction of migration, adhesion, and tissue infiltration of monocytes and polymorphonuclear leukocytes. J Exp Med 180: 203-209, 1994. 
8. Cao QZ and Lin ZB: Antitumor and anti-angiogenic activity of Ganoderma lucidum polysaccharides peptide. Acta Pharmacol Sin 25: 833-838, 2004.

9. Sliva D, Labarrere C, Slivova V, Sedlak M, Lloyd FP Jr and Ho NW: Ganoderma lucidum suppresses motility of highly invasive breast and prostate cancer cells. Biochem Biophys Res Commun 298: 603-612, 2002.

10. Müller CI, Kumagai T, O'Kelly J, Seeram NP, Heber D and Koeffler HP: Ganoderma lucidum causes apoptosis in leukemia, lymphoma and multiple myeloma cells. Leuk Res 30: 841-848, 2006.

11. Kim HS, Kacew S and Lee BM: In vitro chemopreventive effects of plant polysaccharides (Aloe barbadensis miller, Lentinus edodes, Ganoderma lucidum and Coriolus versicolor). Carcinogenesis 20: 1637-1640, 1999.

12. Cheung WM, Hui WS, Chu PW, Chiu SW and Ip NY: Ganoderma extract activates MAP kinases and induces the neuronal differentiation of rat pheochromocytoma PC12 cells. FEBS Lett 486: 291-296, 2000.

13. Pan Y, Song QL, Lin YH, Lu N, Yu HM and Li XJ: GLB prevents tumor metastasis of Lewis lung carcinoma by inhibiting tumor adhesion actions. Acta Pharmacol Sin 26: 881-886, 2005.

14. Urieli-Shoval S, Linke RP and Matzner Y: Expression and function of serum amyloid A, a major acute-phase protein, in normal and disease states. Curr Opin Hematol 7: 64-69, 2000.

15. Christofori G: New signals from the invasive front. Nature 441: 444-450, 2006.

16. Li SQ, Qi HW, Wu CG, Zhang XJ, Yang SG, Zhao X, Wu Z, Wang Y, Que HP and Liu SJ: Comparative proteomic study of acute pulmonary embolism in a rat model. Proteomics 7: 2287-2299, 2007.

17. Gutfeld O, Prus D, Ackerman Z, Dishon S, Linke RP, Levin M and Urieli-Shoval S: Expression of serum amyloid A, in normal, dysplastic and neoplastic human colonic mucosa: implication for a role in colonic tumorigenesis. J Histochem Cytochem 54: 63-73, 2006.

18. Combaret V, Bergeron C, Bréjon S, Iacono I, Perol D, Négrier S and Puisieux A: Protein chip array profiling analysis of sera from neuroblastoma patients. Cancer Lett 228: 91-96, 2005.

19. Chan DC, Chen CJ, Chu HC, Chang WK, Yu JC, Chen YJ, Wen LL, Huang SC, Ku CH, Liu YC and Chen JH: Evaluation of serum amyloid A as a biomarker for gastric cancer. Ann Surg Oncol 14: 84-93, 2007.

20. Niemi K, Baumann MH, Kovanen PT and Eklund KK: Serum amyloid A (SAA) activates human mast cells which leads into degradation of SAA and generation of an amyloidogenic SAA fragment. Biochim Biophys Acta 1762: 424-430, 2006.

21. Van der Westhuyzen DR, Cai L, De Beer MC and De Beer FC: Serum amyloid A promotes cholesterol efflux mediated by scavenger receptor B-I. J Biol Chem 280: 35890-35895, 2005.
22. Preciado-Patt L, Levartowsky D, Prass M, Hershkoviz R, Lider O and Fridkin M: Inhibition of cell adhesion to glycoproteins of the extracellular matrix by peptides corresponding to serum amyloid A. Toward understanding the physiological role of an enigmatic protein. Eur J Biochem 223: 35-42, 1994.

23. Brinckerhoff CE, Mitchell TI, Karmilowicz MJ, KluveBeckerman B and Benson MD: Autocrine induction of collagenase by serum amyloid A-like and beta 2-microglobulinlike proteins. Science 243: 655-657, 1989.

24. Migita K, Kawabe Y, Tominaga M, Origuchi T, Aoyagi T and Eguchi K: Serum amyloid A protein induces production of matrix metalloproteinases by human synovial fibroblasts. Lab Invest 78: 535-539, 1998.

25. Mullan RH, Bresnihan B, Golden-Mason L, Markham T, O'Hara R, FitzGerald O, Veale DJ and Fearon U: Acute-phase serum amyloid A stimulation of angiogenesis, leukocyte recruitment and matrix degradation in rheumatoid arthritis through an NF-kappaB-dependent signal transduction pathway. Arthritis Rheum 54: 105-114, 2006.

26. Yokoi K, Shih LC, Kobayashi R, Koomen J, Hawke D, Li D, Hamilton SR, Abbruzzese JL, Coombes KR and Fidler IJ, Serum amyloid A as a tumor marker in sera of nude mice with orthotopic human pancreatic cancer and in plasma of patients with pancreatic cancer. Int J Oncol 27: 1361-1369, 2005.

27. Khan N, Cromer CJ, Campa M and Patz EF Jr: Clinical utility of serum amyloid A and macrophage migration inhibitory factor as serum biomarkers for the detection of non-small cell lung carcinoma. Cancer 101: 379-384, 2004.

28. Cho WC, Yip TT, Yip C, Yip V, Thulasiraman V, Ngan RK, Yip TT, Lau WH, Au JS, Law SC, Cheng WW, Ma VW and Lim CK: Identification of serum amyloid a protein as a potentially useful biomarker to monitor relapse of nasopharyngeal cancer by serum proteomic profiling. Clin Cancer Res 10: 43-52, 2004

29. Iwamoto Y, Nomizu M, Yamada Y, Ito Y, Tanaka K and Sugioka Y: Inhibition of angiogenesis, tumour growth and experimental metastasis of human fibrosarcoma cells HT1080 by a multimeric form of the laminin sequence Tyr-Ile-Gly-SerArg (YIGSR). Br J Cancer 73: 589-595, 1996.

30. Nicosia RF and Bonanno E: Inhibition of angiogenesis in vitro by Arg-Gly-Asp-containing synthetic peptide. Am J Pathol 138: 829-833, 1991.

31. Chiang HS, Yang RS and Huang TF: The Arg-Gly-Aspcontaining peptide, rhodostomin, inhibits in vitro cell adhesion to extracellular matrices and platelet aggregation caused by saos-2 human osteosarcoma cells. Br J Cancer 71: 265-270, 1995 . 